\title{
Use of MitraClip for Postmyocardial Infarction Mitral Regurgitation Secondary to Papillary Muscle Dysfunction
}

Muhammad Yasin ${ }^{1}$, Aravinda Naniundappa 2 , Frank H. Annie 3 , Alfred Tager 4 , Ali Farooq 5 , Abhishek Bhagat $^{6}$, Vallabh Karpe ${ }^{7}$

1. Internal Medicine, Charleston Area Medical Center, Charleston , USA 2. Cardiology, Charleston Area Medical Center, Bowie, USA 3. Cardiology, Charleston Area Medical Center/Health Education and Research Institute, Charleston, USA 4. Emergence Medicine, Charleston Area Medical Center, Charleston, USA 5. Cardiology, Charleston Area Medical Center, Charleston, USA 6. Internal Medicne, Charleston Area Medical Center, Charleston, USA 7. CAMC, Charleston Area Medical Center, Charleston, USA

Corresponding author: Frank H. Annie, frank.h.annie@camc.org

\section{Abstract}

Papillary muscle rupture after acute myocardial infarction (AMI) is a dreadful complication and it is associated with five percent of deaths following AMI. Surgery is the recommended treatment of choice; however, it is usually deferred due to the high risk of mortality. MitraClip implantation using a transcatheter approach is an alternative option for patients with severe mitral regurgitation (MR) following AMI or those with high operative risk. We report a case of a 68-year-old male patient who developed severe MR secondary to AMI and underwent successful mitral valve repair using the MitraClip device.

Categories: Cardiac/Thoracic/Vascular Surgery, Cardiology

Keywords: mitraclip, myocardial infarction, regurgitation

\section{Introduction}

Flail mitral valve (MV) leaflet after acute myocardial infarction (AMI) is a lethal complication associated with five percent mortality post-AMI [1-2]. MV repair showed a better outcome than replacement in terms of mortality, survival, ventricular function preservation, and risk of endocarditis for most cases. MitraClip implantation using a transcatheter approach is an alternative option for patients with severe mitral regurgitation (MR) due to flail MV following AMI who have a high operative risk [3]. The most effective current technique for MV repair is the double orifice MV configuration. Applying that technique percutaneously using the MitraClip system can result in lower mortality, morbidity, and recurrence of mitral regurgitation (MR) in most patients. We report a case of a 68 -year-old male patient who developed severe MR secondary to AMI and underwent successful MV repair using the MitraClip device.

Received 06/28/2018

Review began 07/19/2018 Review ended 07/25/2018 Published 07/30/2018

๑) Copyright 2018

Yasin et al. This is an open access article distributed under the terms of the Creative Commons Attribution License CC-BY 3.0., which permits unrestricted use, distribution, and reproduction in any medium, provided the original author and source are credited.

\section{Case Presentation}

A 68-year-old Caucasian male with a history of hypertension, hypercholesterolemia, coronary artery disease status post five vessel coronary artery bypass grafting presented to the emergency room with chest pain for four days which radiated to his jaw and along the left arm (Figure 1).

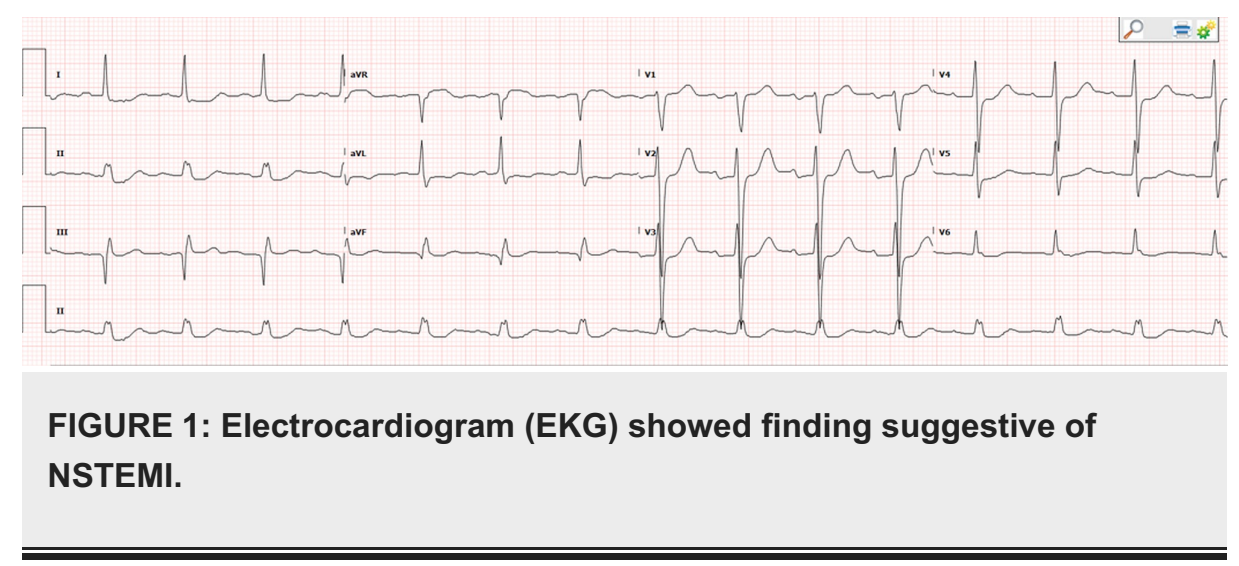

Electrocardiogram (EKG) showed old Q waves in inferior leads and new ST depression in leads I, AVL, V5 and V6 and troponin peaked up to 15.8. At the time of presentation, the patient was also short of breath with initial chest plain, a radiograph (X-ray) showing pulmonary edema is depicted (Figures 2 and 3 ). 


\section{Cureus}

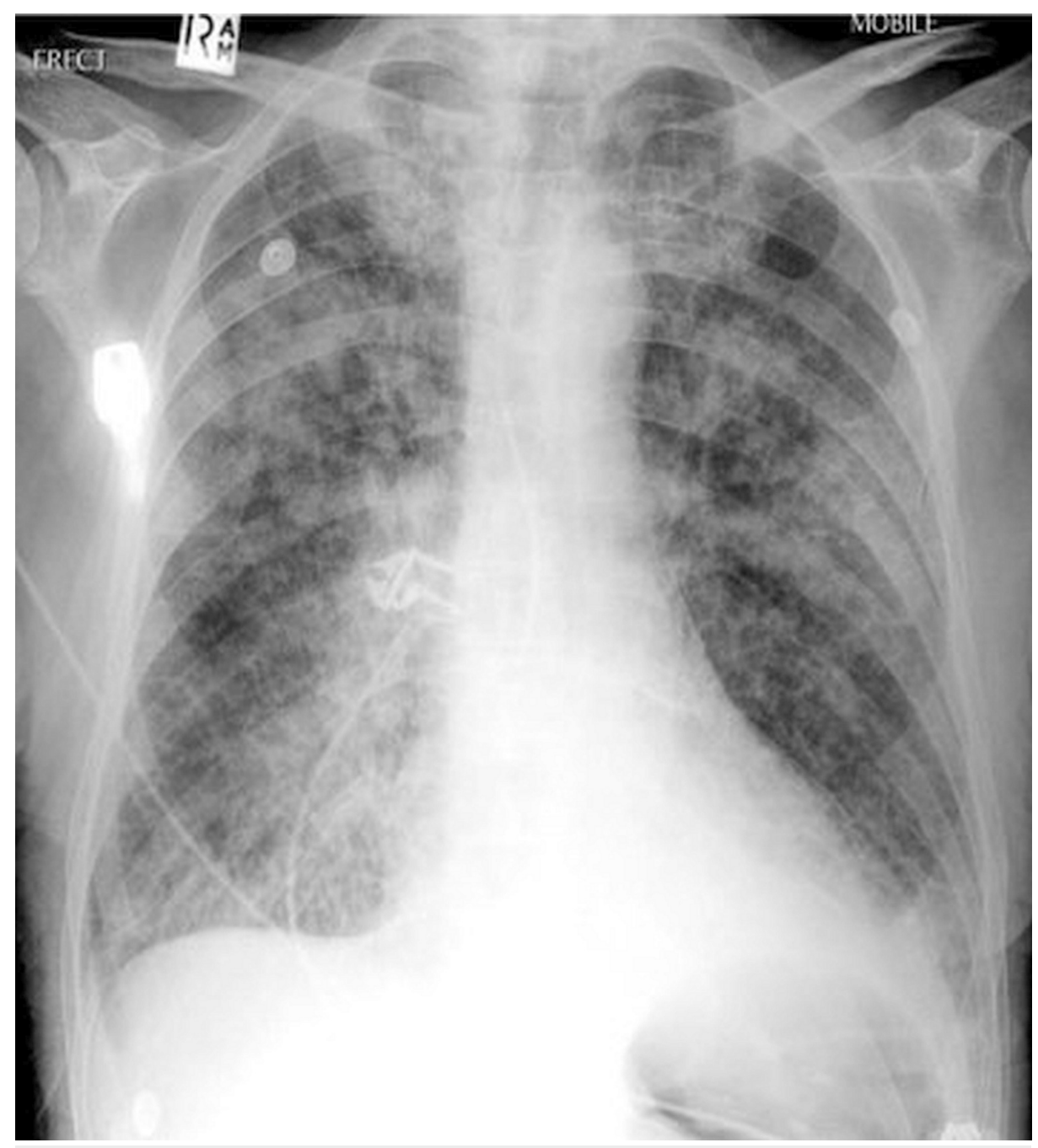

FIGURE 2: Chest X-ray showing pulmonary edema. 


\section{Cureus}

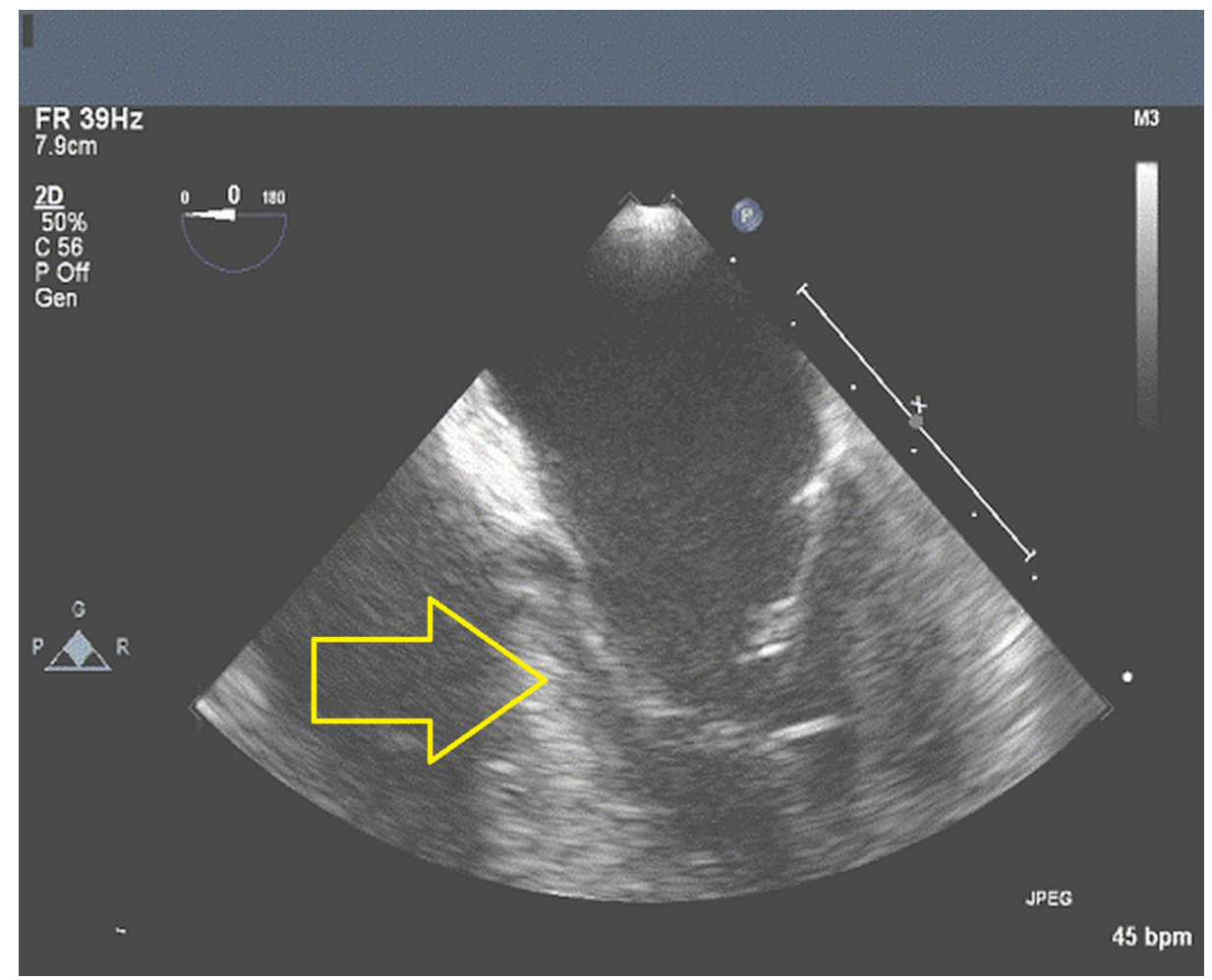

FIGURE 3: Echocardiography (ECHO) showed partial disruption of medial papillary muscle with severely flail posterior mitral valve (MV) leaflet and severe mitral regurgitation (MR).

The patient was electively intubated before urgent cardiac catheterization, which was performed within 12 hours of patient's arrival, and revealed $100 \%$ occlusion in right coronary, left main coronary, and the proximal portion of the circumflex artery as well as diffuse disease of the grafted vessels to circumflex. Furthermore, during catheterization, the patient was also noted to have hemodynamic compromise with blood pressure observed to be $86 / 63 \mathrm{mmHg}$ and a heart rate of 115 beats per minute, which prompted emergency echocardiography (ECHO) showing partial disruption of medial papillary muscle with severely flail posterior MV leaflet and severe MR. He subsequently underwent venoarterial extracorporeal membrane oxygenation (ECMO) pump placement by cardiothoracic surgery for hemodynamic support accessing through the right common femoral artery and right femoral vein (Figure 4). 


\section{Cureus}

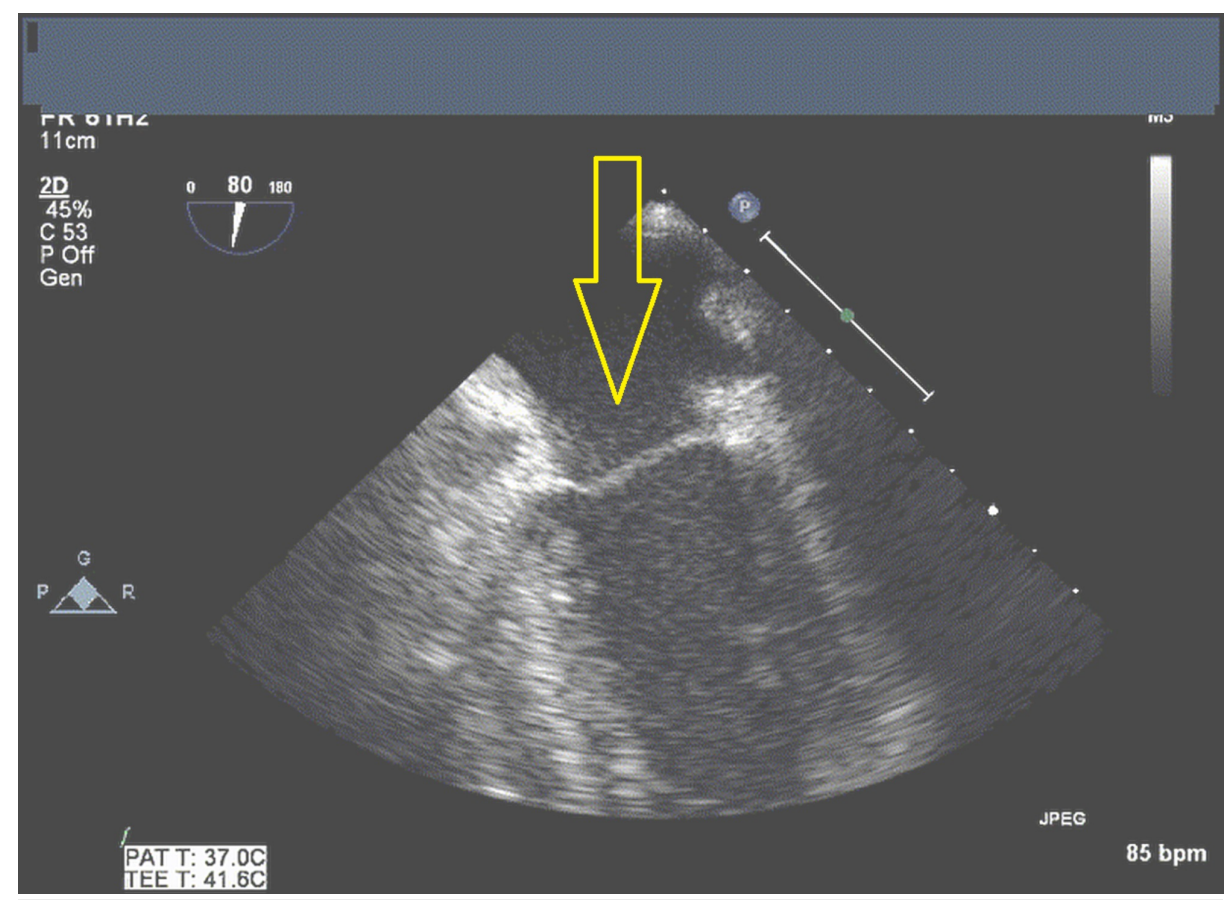

FIGURE 4: Flail motion due to rupture of the posteromedial papillary muscle head. There is severe regurgitation, directed eccentrically.

Heart team specialists thus discussed the possibility of performing an MV clipping, and it was decided in favor of proceeding with the transcatheter procedure as the patient had a history of median sternotomy and previous bypass surgery. The patient remained intubated and was taken to cath lab on the fifth day of hospitalization. The femoral vein was accessed using ultrasound guidance, and the transseptal puncture was performed using intraoperative transesophageal echocardiography. This was then followed by prepping the MitraClip using standard technique and positioning it across the interatrial septum and above the atrial valve. For this procedure, two clips were used, one was on the posterior segment $\mathrm{P} 2$ and just beside it to cinch up P2 and A2, and the second one for P2 and P1 laterally. Once the resulting MR reduction was regarded as adequate, clips were deployed (Figures 5 and 6).

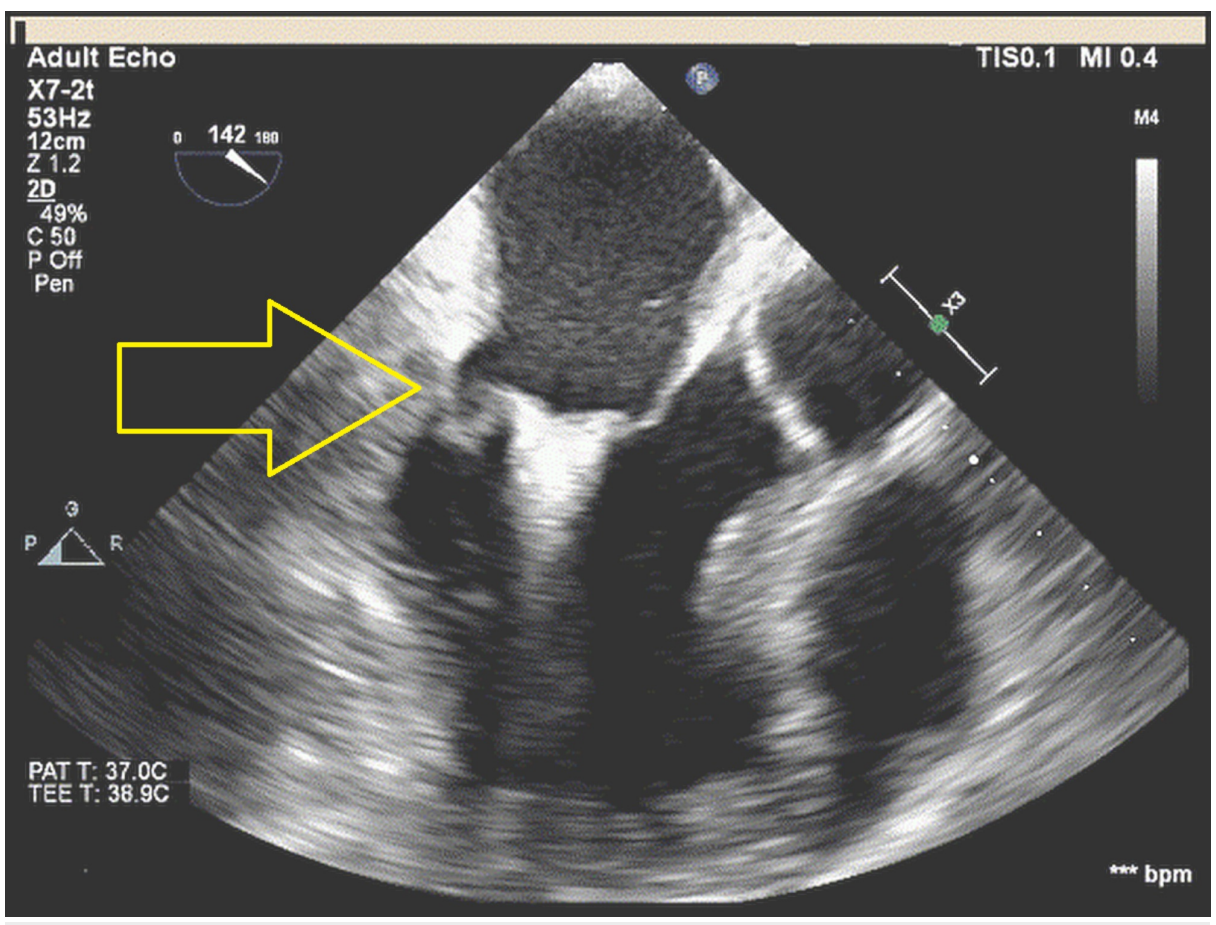

FIGURE 5: Femoral vein was accessed using ultrasound guidance and 
transseptal puncture was performed using intraoperative transesophageal echocardiography (ECHO). This was then followed by prepping the MitraClip using standard technique and positioning it across the interatrial septum and above the atrial valve. For this procedure, two clips were used, one was on the posterior segment P2 and just beside it to cinch up P2 and A2, and the second one for P2 and $\mathrm{P} 1$ laterally. Once the resulting mitral regurgitation (MR) reduction was regarded adequate, clips were deployed.

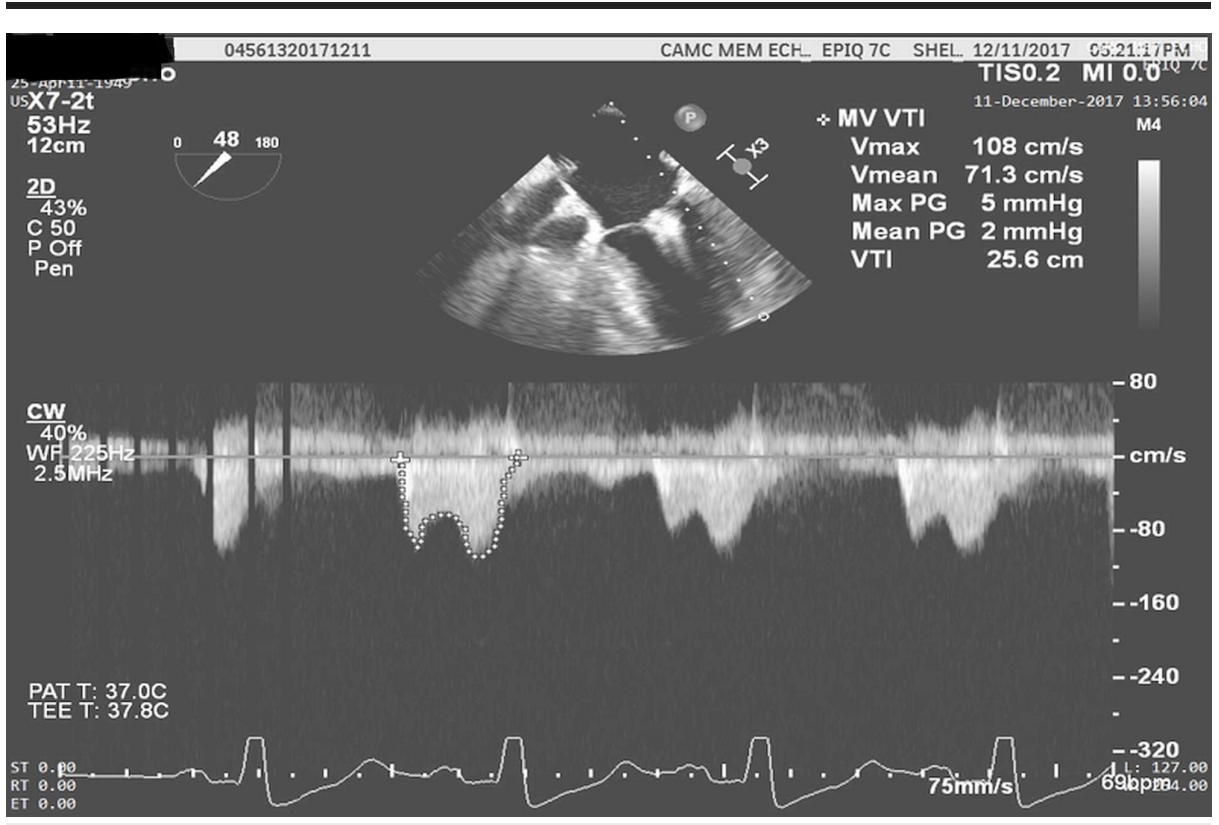

FIGURE 6: Intraoperative transesophageal echocardiography (ECHO).

The only complication noted was the transient Wenckebach heart block for which the patient subsequently received a pacemaker later in the hospital course. Thus, the patient underwent two successful MitraClip placements, and the severity of MR went down from $4+$ to no more than $1+$. The patient's left ventricular function improved postprocedure and ECMO was subsequently followed by extubation later on. Repeat ECHO on third postprocedure day showed mild MR and normal left ventricular ejection fraction. At the 30th day, the patient was followed up in the clinic and repeat ECHO at that time showed mild MR only.

\section{Discussion}

Acute myocardial infarction with associated papillary muscle dysfunction leads to MR, and concomitant cardiogenic shock carries high mortality risk and accounts for approximately $5 \%$ of deaths following AMI [1]. The most common culprit vessel is the right coronary artery, but the condition can involve the left circumflex artery as well, albeit less commonly [4]. Our case involved both the right coronary and left circumflex arteries. Pulmonary edema or signs and symptoms of cardiogenic shock in the following AMI should prompt urgent investigation. Once this catastrophe is identified, medical treatment is limited and associated with poor outcomes [5]. The standard therapy is surgical repair or replacement, a class I recommendation in severe MR [2]. Despite the decreased incidence of perioperative mortality associated with surgery, there is still a substantial risk associated with this procedure [6]. Prior to receiving this definitive treatment, patients require supportive treatment with vasodilator therapy and interval hemodynamic support using an intra-aortic balloon pump or ECMO [1]. We used venoarterial ECMO to provide hemodynamic support for our patient. Percutaneous transcatheter MV repair using the MitraClip is an emerging alternative option for patients with severe MR who are considered as high risk for open repair [3]. The common indications for the MitraClip procedure for MR repair include chronic degenerative or functional etiologies, and data on MitraClup use in acute settings are scarce. Nevertheless, both etiologies can present in the setting of AMI [7]. Degenerative MR is caused by an abnormality in the structural apparatus of the MV whereas functional MR occurs without any structural deformity. To our knowledge, ours is the 10th reported case of a mitral clip used in an acute setting. Our patient's severe MR was caused by posterior papillary muscle rupture as a complication of AMI. Currently, there are reservations for the use of MitraClip in the setting of AMI given the risk of papillary rupture with leaflet detachment and the lack of 
sufficient data in support of (and possible improvement of) MR severity following revascularization once the myocardium is revitalized [8]. The MitraClip consists of two arms that open and close using delivery system operated by an expert in structural heart disease [9-10]. Once approximated at the leaflet margin, the system is deployed ensuring a reduction in the severity of MR under fluoroscopic and transesophageal echocardiographic guidance.

\section{Conclusions}

Our case represents a successful treatment with a MitraClip using the double orifice technique and suggests that this minimally invasive technique may be useful for high-risk surgical candidates with cardiogenic shock and pulmonary edema following AMI. It also shows that using this technique percutaneously might result in lower mortality rate, higher survival rate, with a lower risk of endocarditis following the procedure. Additional studies are necessary to establish the long-term efficacy and safety of this procedure. Due to a lack of data, a multidisciplinary team consisting of experts in structural heart disease should make the decision based on risk assessment scores, clinical presentation, morphology of MV, and cardiac function. Our case represents a successful treatment involving a MitraClip using the double orifice technique and suggests this minimally invasive technique may be useful for high-risk surgical candidates with cardiogenic shock and pulmonary edema following AMI. It also shows that using this technique percutaneously might result in lower mortality rate, higher survival rate, with a lower risk of endocarditis following the procedure. Additional studies are necessary to establish the long-term efficacy and safety of this procedure. Due to a lack of data, a multidisciplinary team consisting of experts in structural heart disease should make the decision based on risk assessment scores, clinical presentation, morphology of MV, and cardiac function.

\section{Additional Information \\ Disclosures}

Human subjects: Consent was obtained by all participants in this study. Conflicts of interest: In compliance with the ICMJE uniform disclosure form, all authors declare the following: Payment/services info: All authors have declared that no financial support was received from any organization for the submitted work. Financial relationships: All authors have declared that they have no financial relationships at present or within the previous three years with any organizations that might have an interest in the submitted work. Other relationships: All authors have declared that there are no other relationships or activities that could appear to have influenced the submitted work.

\section{References}

1. Nishimura RA, Schaff HV, Shub C, Gersh BJ, Edwards WD, Tajik AJ: Papillary muscle rupture complicating acute myocardial infarction: analysis of 17 patients. Am J Cardiol. 1983, 51:373-377. 10.1016/S00029149(83)80067-8

2. Bonow RO, Carabello BA, Chatterjee K, et al.: 2008 focused update incorporated into the ACC/AHA 2006 guidelines for the management of patients with valvular heart disease: a report of the American College of Cardiology/American Heart Association Task Force on Practice Guidelines (Writing Committee to revise the 1998 guidelines for the management of patients with valvular heart disease) Endorsed by the Society of Cardiovascular Anesthesiologists, Society for Cardiovascular Angiography and Interventions, and Society of Thoracic Surgeons. J Am Coll Cardiol. 2008, 52:1-142. 10.1016/j.jacc.2008.05.007

3. O'Gara PT, Calhoon JH, Moon MR: Transcatheter therapies for mitral regurgitation: a professional society overview from the American College of Cardiology, the American Association for Thoracic Surgery, Society for Cardiovascular Angiography and Interventions Foundation, and the Society of Thoracic Surgeons. Catheter Cardiovasc Interv. 2014, 83:849-863. 10.1002/ccd.25306

4. Figueras J, Calvo F, Cortadellas J, Soler-Soler J: Comparison of patients with and without papillary muscle rupture during acute myocardial infarction. Am J Cardiol. 1997, 80:625-627. 10.1016/S0002-9149(97)004359

5. Thompson CR, Buller CE, Sleeper LA, et al.: Cardiogenic shock due to acute severe mitral regurgitation complicating acute myocardial infarction: a report from the SHOCK Trial Registry. J Am Coll Cardiol. 2000, 36:1104-1109. 10.1016/S0735-1097(00)00846-9

6. Russo A, Suri RM, Grigioni F, et al.: Clinical outcome after surgical correction of mitral regurgitation due to papillary muscle rupture. Circulation. 2008, 118:1528-1534. 10.1161/CIRCULATIONAHA.107.747949

7. Kumanohoso T, Otsuji Y, Yoshifuku S, et al.: Mechanism of higher incidence of ischemic mitral regurgitation in patients with inferior myocardial infarction: quantitative analysis of left ventricular and mitral valve geometry in 103 patients with prior myocardial infarction. J Thorac Cardiovasc Surg. 2003, 125:135-143.

8. Tarsia G, Smaldone C, Costantino MF: Effective percutaneous "edge-to-edge" mitral valve repair with mitraclip in a patient with acute post-MI regurgitation not related to papillary muscle rupture. Catheter Cardiovasc Interv. 2016, 88:1177-1180. 10.1002/ccd.26416

9. Alfieri O, Maisano F, De Bonis M, et al.: The double-orifice technique in mitral valve repair: a simple solution for complex problems. J Thorac Cardiovasc Surg. 2001, 122:674-681. 10.1067/mtc.2001.117277

10. Feldman T, Kar S, Rinaldi M, et al.: Percutaneous mitral repair with the MitraClip system: safety and midterm durability in the initial EVEREST (Endovascular Valve Edge-to-Edge REpair Study) cohort. J Am Coll Cardiol. 2009, 54:686-694. 10.1016/j.jacc.2009.03.077 